\title{
Generalized parton distributions from domain wall valence quarks and staggered sea quarks
}

\author{
LHPC Collaboration: D.B. Renner, ${ }^{*}{ }^{\dagger}$ J. Bratt, ${ }^{b}$ R.G. Edwards, ${ }^{c}$ M. Engelhardt, ${ }^{d}$ \\ G. Fleming, ${ }^{e}$ Ph. Hägler, ${ }^{f}$ B. Musch, ${ }^{f}$ J.W. Negele, ${ }^{b}$ K. Orginos, ${ }^{c g}$ A.V. Pochinsky, ${ }^{b}$ \\ D.G. Richards, ${ }^{c}$ W. Schroers ${ }^{h}$ \\ ${ }^{a}$ University of Arizona, Department of Physics, 1118 E 4th St, Tucson AZ 85721, USA \\ ${ }^{b}$ Centerfor Theoretical Physics, Massachusetts Institute of Technology, Cambridge, MA 02139, USA \\ ${ }^{c}$ Thomas Jefferson National Accelerator Facility, Newport News, VA 23606, USA \\ ${ }^{d}$ Physics Department, New Mexico State University, Las Cruces, NM 88003-8001 \\ e Sloane Physics Laboratory, Yale University, New Haven, CT 06520, USA \\ ${ }^{f}$ Institut für Theoretische Physik, TU München, D-85747 Garching, Germany \\ ${ }^{g}$ Department of Physics, College of William and Mary, Williamsburg VA 23187, USA \\ ${ }^{h}$ John von Neumann-Institut für Computing NIC/DESY, D-15738 Zeuthen, Germany
}

\begin{abstract}
Moments of the generalized parton distributions of the nucleon, calculated with a mixed action of domain wall valence quarks and asqtad staggered sea quarks, are presented for pion masses extending down to $359 \mathrm{MeV}$. Results for the moments of the unpolarized, helicity, and transversity distributions are given and compared to the available experimental measurements. Additionally, a selection of the generalized form factors are shown and the implications for the spin decomposition and transverse structure of the nucleon are discussed. Particular emphasis is placed on understanding systematic errors in the lattice calculation and exploring a variety of chiral extrapolations.
\end{abstract}

The XXV International Symposium on Lattice Field Theory

July 30 - August 42007

Regensburg, Germany

\footnotetext{
* Speaker.

${ }^{\dagger}$ Current address: DESY Zeuthen, Platanenallee 6, D-15738 Zeuthen, Germany
} 
Lattice / Experiment

\begin{tabular}{|l|l|l|l|l|}
\hline$a m_{u / d}^{\text {asqtad }}$ & $L / a$ & $L$ & $m_{\pi}^{\text {DWF }}$ & $\#$ \\
\hline & & $\mathrm{fm}$ & $\mathrm{MeV}$ & \\
\hline 0.05 & 20 & 2.52 & 761 & 425 \\
\hline 0.04 & $”$ & $"$ & 693 & 350 \\
\hline 0.03 & $”$ & $"$ & 594 & 564 \\
\hline 0.02 & $"$ & $"$ & 498 & 486 \\
\hline 0.01 & $"$ & $"$ & 354 & 656 \\
\hline 0.01 & 28 & 3.53 & 353 & 270 \\
\hline
\end{tabular}

Figure 1: Lattice parameters used in this work.

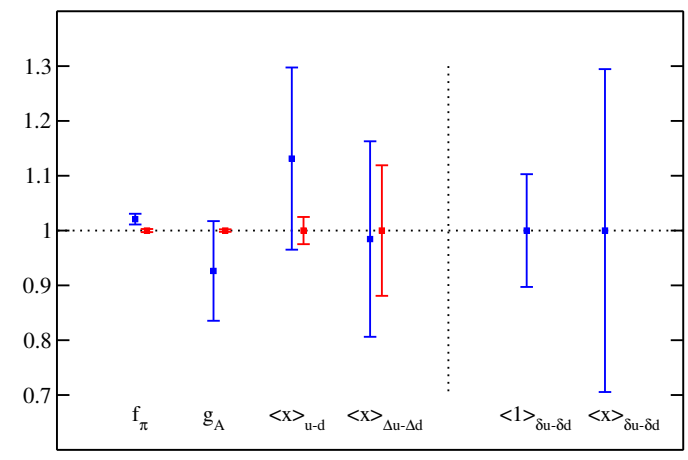

Figure 2: Moments of parton distributions.

\section{Introduction}

Lattice field theory provides a precise definition of nucleon structure observables as well as a numerical means for evaluating them non-perturbatively. Here we focus on the nucleon generalized parton distributions. This set of observables contains the parton distributions and form factors as well as quantities that determine the transverse distribution of quarks within the nucleon and the decomposition of the nucleon spin into quark and gluon degrees of freedom. To perform these calculations, we use a mixed action consisting of domain wall valence quarks on improved staggered sea quark configurations provided by the MILC collaboration [1]. The details of this work have been published in Refs. [2], [3], and [4] and are briefly summarized in Fig. [1]. In this proceeding, we examine the systematic errors related to the determination of matrix elements from lattice correlation functions as well as the extrapolation of lattice results to the physical quark masses.

\section{Moments of Parton Distributions}

As theorists improve algorithms and as the computing resources dedicated to lattice QCD calculations continue to grow, we can begin to contemplate precise calculations of the low nonsinglet moments of the nucleon parton distributions. This will require control of a variety of sources of error. The calculation of any observable requires a study of the volume, lattice spacing, and quark mass dependence. Additional sources of error arise depending on the observable and choice of action. For the calculation of moments of parton distributions, the additional errors are caused by matching correlation functions calculated on the lattice to functional forms derived or motivated by transfer matrix arguments as well as errors due to the renormalization of the operators themselves. Our previous work examined the volume and quark mass dependence of the axial charge [5] and the quark mass dependence of all the low moments [4] and the generalized form factors [6].

\subsection{Correlation Functions}

Simple lattice actions admit a transfer matrix formalism, which allows one to rigorously relate lattice correlation functions and quantum mechanical matrix elements. Domain wall fermions have a transfer matrix in the full five-dimensional space, however, that does not guarantee positive 


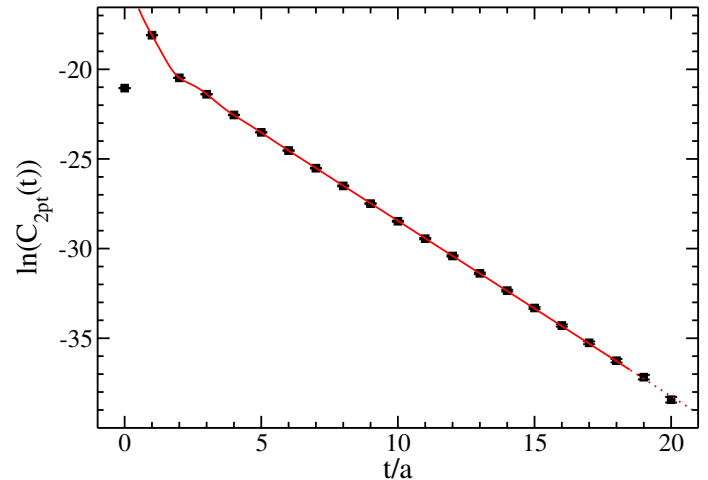

Figure 3: Nucleon two-point correlator and fit.

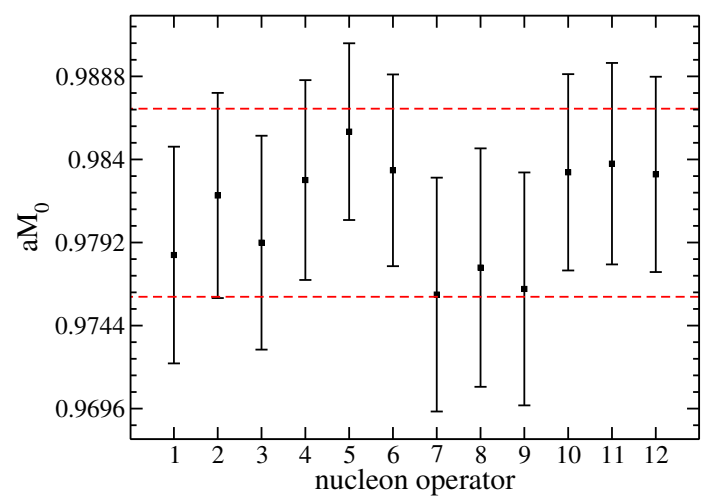

Figure 5: Ground state mass for all fits.

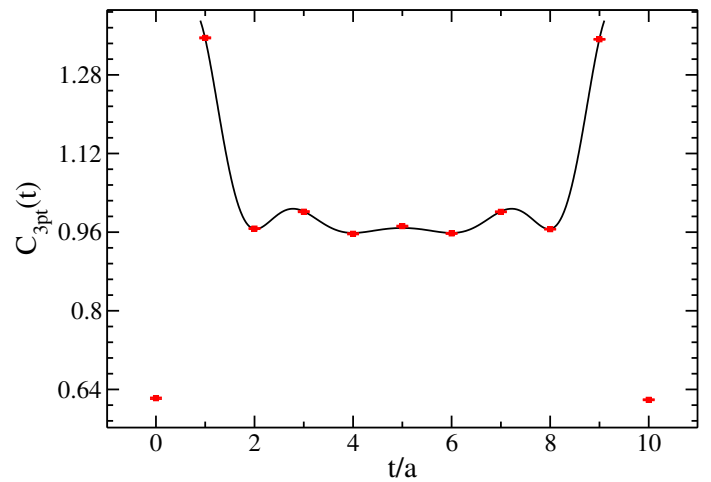

Figure 4: Nucleon three-point plateau and fit.

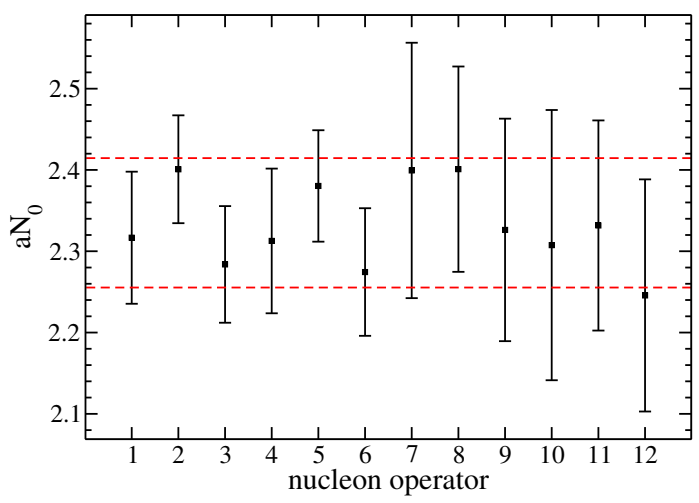

Figure 6: Lowest oscillating mass for all fits.

definite correlation functions in four dimensions. ${ }^{1}$ This results in clear oscillations in correlation functions, as shown in Figs. [3] and [4]. Lacking a theoretical derivation of an effective fourdimensional transfer matrix, we consider the following phenomenological forms, which include the standard positive-definite contributions as well as oscillating contributions.

$$
\begin{aligned}
& C_{2 \mathrm{pt}}(t)=A_{0} \exp \left(-M_{0} t\right)+A_{1} \exp \left(-M_{1} t\right)+B_{0}(-1)^{t} \exp \left(-N_{0} t\right) \\
& C_{3 \mathrm{pt}}(t)=O_{00}+O_{10} \cosh \left(\left(M_{1}-M_{0}\right) t\right)+P_{00}(-1)^{t} \cosh \left(\left(N_{0}-M_{0}\right) t\right)
\end{aligned}
$$

Figures [3] and [4] show fits to Eqs. [2.1] and [2.2], respectively, for the case $m_{\pi}=761 \mathrm{MeV}$. To test this functional form, we fit 12 nucleon two-point correlation functions. The resulting ground state and lowest oscillating state masses are shown in Figs. [5] and [6] respectively, again for $m_{\pi}=761 \mathrm{MeV}$. There is clear agreement for $M_{0}, N_{0}$, and $M_{1}$ (not shown) for each nucleon channel considered, indicating universal values for the physical masses, as expected, and also for the oscillating mass. Therefore we employ this fitting strategy for all the moments of parton distributions presented here and will extend this method to the generalized form factors in a future work.

\footnotetext{
${ }^{1}$ Mixed action calculations in general suffer from a lack of unitarity, despite the choice of valence action.
} 


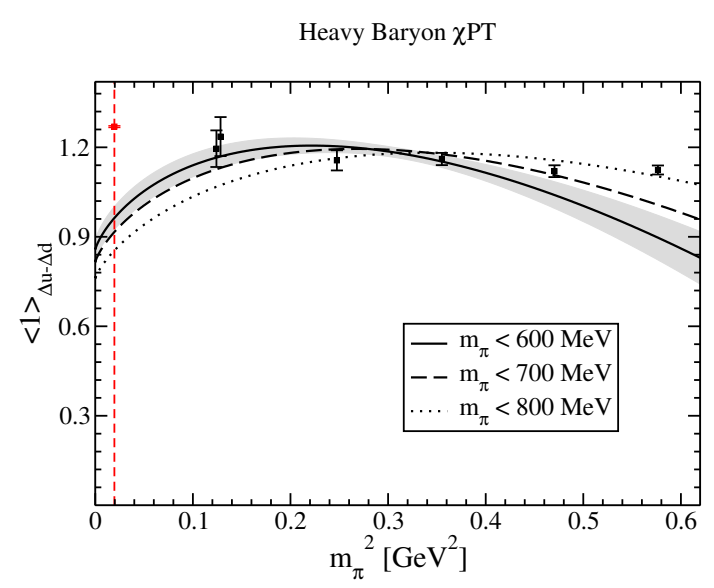

Figure 7: $g_{A}$ and heavy baryon fit.

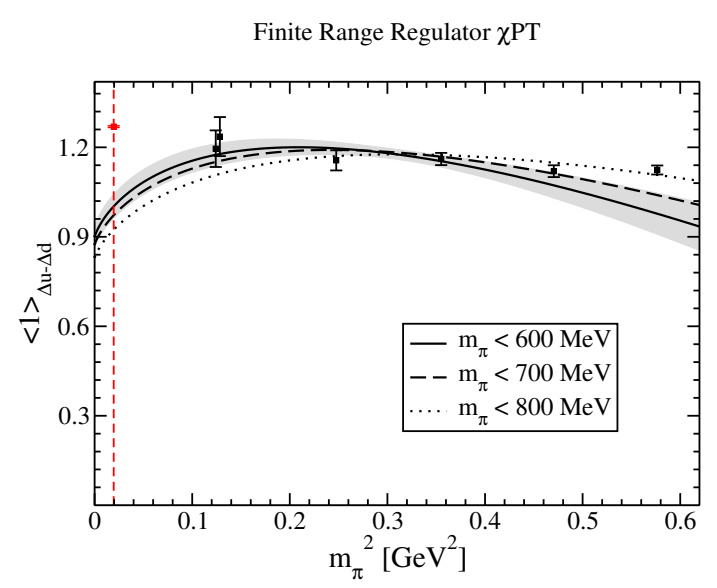

Figure 8: $g_{A}$ and finite range regulator fit.

\subsection{Chiral Perturbation Theory}

There is a variety of methods to perform chiral extrapolations of moments of parton distributions, which should all agree at light enough quark masses, but will, however, systematically differ when applied at the quark masses currently used in lattice calculations. Lattice calculations at the physical quark masses will of course eliminate the need for chiral extrapolations, however, in the interim, we examine a variety of these methods with the ultimate goal of systematically comparing all such methods.

The axial charge and the pion decay constant are low energy constants that are common to all chiral peturbation theory expressions for the moments of parton distributions. Therefore any successful extrapolation method must account for both observables. First we examine two methods that are known to fail. The first is standard heavy baryon chiral perturbation theory and the second is the same expression but with a finite range regulator [7]. Fits to the axial charge for each are shown in Figs. [7] and [8]. The figures show the error band for the most conservative fit, including just the lightest three pion masses. There is a clear discrepancy between the experimental result and the extrapolated lattice result. Additionally, varying the fit range from $m_{\pi}<600 \mathrm{MeV}$ to $m_{\pi}<800 \mathrm{MeV}$ shows a systematic variation.

The above results indicate that the simplest chiral perturbation theory expressions fail to converge for pion masses in the range considered here. A common alternative is to include the Delta in the effective theory and this has been shown to successfully describe the axial coupling in Refs. [5] and [8]. Here we describe a simpler alternative [4] that seems to correctly reproduce the experimental values for $g_{A}, f_{\pi}$, and other low moments of parton distribution functions. We start with the standard chiral perturbation expressions for the various moments of parton distributions $[9,10]$. The renormalization scale $\mu$ is eliminated in favor of a dimensionless quantity, $\alpha$, by setting $\mu=\alpha f$. ${ }^{2}$ The values of $g_{A}$ and $f_{\pi}$ in the chiral limit occur in the expressions for the moments, but always in the next-to-leading-order (NLO) term. In order to eliminate the need for multiple combined fits, we can eliminate the chiral limit values of all observables that occur in the NLO terms in favor of

\footnotetext{
${ }^{2}$ The chiral limit values of $g_{A}, f_{\pi}$, and moments like $\langle x\rangle$ are denoted by $\stackrel{\circ}{g}, \stackrel{\circ}{f}$, and $\langle\stackrel{\circ}{x}\rangle$. Additionally, explicit labels of $\pi$ are dropped in Eqs. [2.3,2.4,2.5].
} 


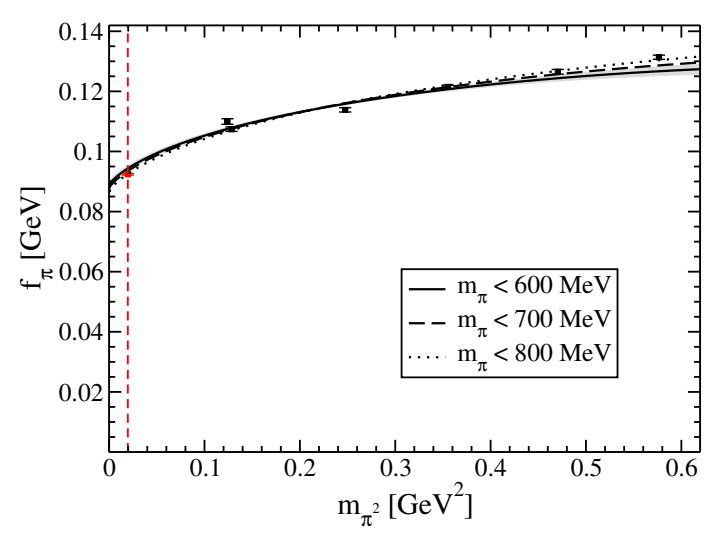

Figure 9: $f_{\pi}$ and self-consistent fit.

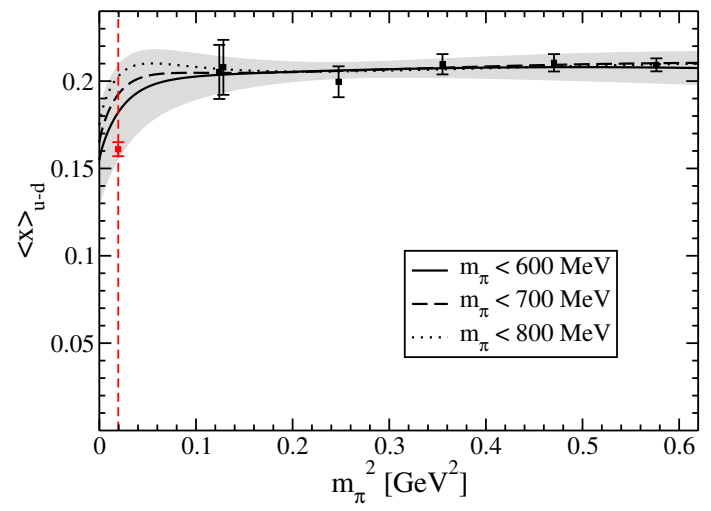

Figure 11: $\langle x\rangle_{u-d}$ and self-consistent fit.

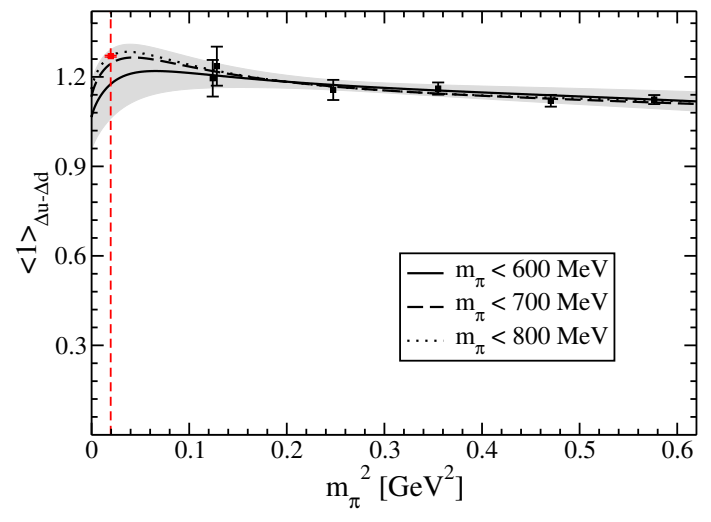

Figure 10: $g_{A}$ and self-consistent fit.

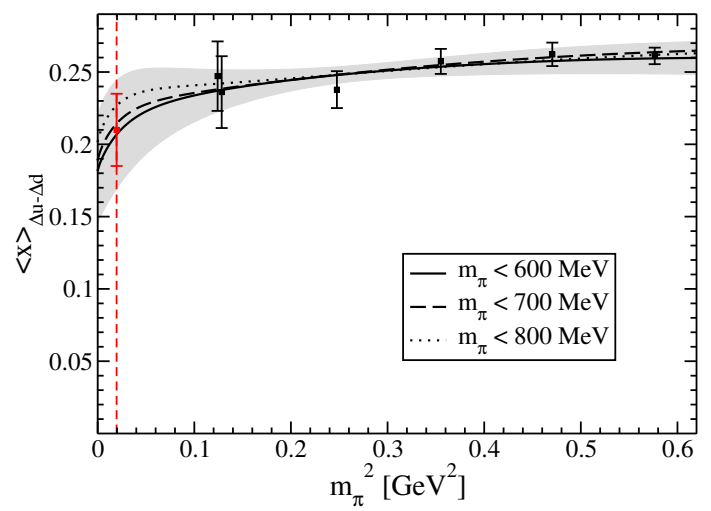

Figure 12: $\langle x\rangle_{\Delta u-\Delta d}$ and self-consistent fit.

their lattice values at the corresponding pion mass. ${ }^{3}$ This simplifies the fits and does not change the chiral expressions to the order at which we are working. The resulting expressions are given below.

$$
\begin{aligned}
\langle x\rangle\left(1+\left(3 g^{2}+1\right) m^{2} /(4 \pi f)^{2} \ln \left(m^{2} /(\alpha f)^{2}\right)\right) & =\langle\stackrel{\circ}{x}\rangle+c_{x}(\alpha) m^{2} \\
g\left(1+\left(2 g^{2}+1\right) m^{2} /(4 \pi f)^{2} \ln \left(m^{2} /(\alpha f)^{2}\right)\right) & =\stackrel{\circ}{g}+c_{g}(\alpha) m^{2} \\
f\left(1+m^{2} /(4 \pi f)^{2} \ln \left(m^{2} /(\alpha f)^{2}\right)\right) & =\stackrel{\circ}{f}+c_{f}(\alpha) m^{2}
\end{aligned}
$$

Each of Eqs. [2.3,2.4,2.5] is an independent simple linear fit for a chiral limit value and a counterterm. To plot a moment as a smooth curve that corresponds to these fits, one first solves the transcendental Eq. [2.5] for $f$, then the cubic equation Eq. [2.4] for $g$, and then the linear Eq. [2.3] for $\langle x\rangle$. As examples, the resulting fits for $f_{\pi}, g_{A},\langle x\rangle_{u-d}$, and $\langle x\rangle_{\Delta u-\Delta d}$ are shown in Figs. [9-12]. In each case we find agreement, within the statistical errors, between the chiral extrapolation and the experimental measurement. In Fig. [2] we collect these results along with the lowest transversity moments in order to highlight the genuine potential for predictions of transversity distributions from lattice QCD calculations. In this figure, we normalize each result to the experimental measur-

\footnotetext{
${ }^{3}$ For similar ideas, see also Refs. [11, 12, 13, 14, 15, 16, 17, 18].
} 


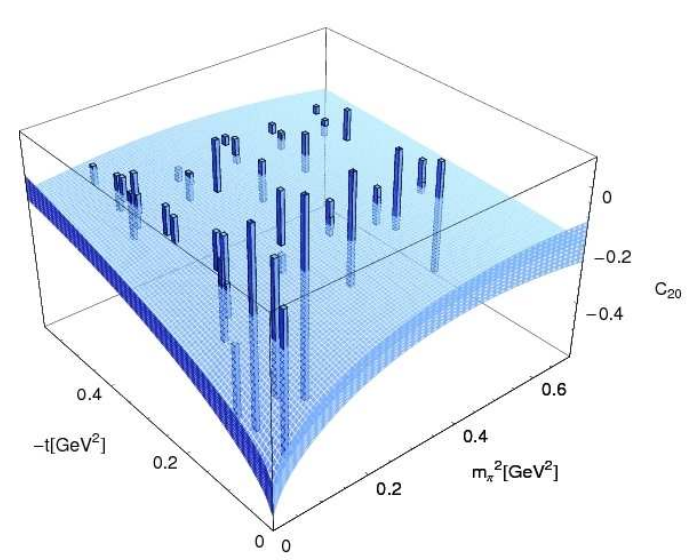

Figure 13: $C_{20}(t)$ and heavy baryon fit.

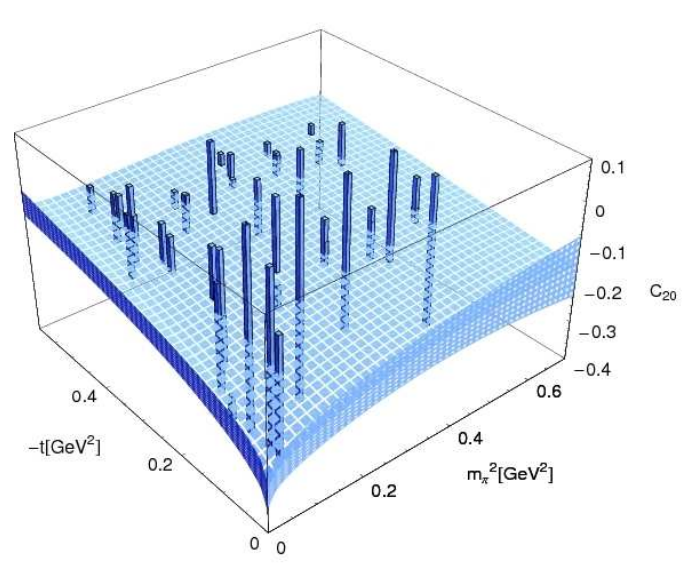

Figure 14: $C_{20}(t)$ and covariant fit.

ment where available; otherwise we normalize by the lattice calculation. For each observable, the leftmost point is the lattice calculation and the rightmost point is the experimental measurement.

\section{Generalized Form Factors}

The low non-singlet moments of the nucleon parton distributions serve as benchmark calculations: as the precision for the moments increases, so does the confidence in the results for the generalized form factors. Extensive results relating to the transverse structure and spin decomposition as well as several comparative studies of chiral perturbation are given in Ref. [6]. Here we focus on one example of the application of chiral perturbation theory to the generalized form factor, $C_{20}$. As for the parton distributions, there are several varieties of effective field theory methods available to extrapolate both the pion mass and momentum, $t$, dependence of generalized form factors. Figures [13] and [14] show our results for the $C_{20}$ form factor and the fits to heavy baryon [19, 20] and covariant [21] chiral perturbation theory. These results illustrate again the benefits of examining multiple methods of chiral extrapolations.

\section{Conclusions}

Given the scale of computing resources estimated in the near future, it is likely that the simplest aspects of nucleon structure will be calculated with quantitatively controlled errors. This will require a concerted effort to control all sources of errors in our calculations. We examine here the traditional sources of error associated with extracting matrix elements from lattice correlation functions as well as several varieties of chiral extrapolation methods. Though the simplest methods still fail to correctly accomodate the current lattice results and reproduce the experimental measurements, we find that the self-consistent replacement of the chiral limit values of $g_{A}, f_{\pi}$, and the moments $\left\langle x^{n}\right\rangle$ with the correpsonding lattice results appears to improve convergence in all cases that can be compared with experimental results. Additionally, we illustrate the potential to examine not only the pion mass dependence but also the momentum dependence of form factors using chiral perturbation theory. 


\section{Acknowledgments}

This work was supported by the DOE Office of Nuclear Physics under contracts DE-FC0294ER40818, DE-AC05-06OR23177 and DE-AC05-84150, the EU Integrated Infrastructure Initiative Hadron Physics (I3HP) under contract RII3-CT-2004-506078, the DFG under contract FOR 465 (Forschergruppe Gitter-Hadronen-Phänomenologie) and the DFG Emmy-Noether program. Computations were performed on clusters at Jefferson Laboratory and at ORNL using time awarded under the SciDAC initiative. We are indebted to the members of the MILC collaboration for providing the dynamical quark configurations which made our full QCD calculations possible.

\section{References}

[1] C. W. Bernard et al., Phys. Rev. D64, 054506 (2001).

[2] D. B. Renner et al., Nucl. Phys. Proc. Suppl. 140, 255 (2005).

[3] R. G. Edwards et al., PoS LAT2005, 056 (2006).

[4] R. G. Edwards et al., PoS LAT2006, 121 (2006).

[5] R. G. Edwards et al., Phys. Rev. Lett. 96, 052001 (2006).

[6] Ph. Hägler et al., arXiv:0705.4295 [hep-lat] (2007).

[7] W. Detmold et al., Phys. Rev. Lett. 87, 172001 (2001).

[8] A. Ali Khan et al., Phys. Rev. D74, 094508 (2006).

[9] D. Arndt and M. J. Savage, Nucl. Phys. A697, 429 (2002).

[10] J. W. Chen and X. D. Ji, Phys. Lett. B523, 107 (2001).

[11] S. R. Beane et al., Phys. Rev. D73, 054503 (2006).

[12] J. W. Chen, D. O'Connell, R. S. Van de Water, and A. Walker-Loud, Phys. Rev. D73, 074510 (2006).

[13] D. O'Connell, hep-lat/0609046 (2006).

[14] S. R. Beane et al., Phys. Rev. D74, 114503 (2006).

[15] S. R. Beane et al., Phys. Rev. D75, 094501 (2007).

[16] S. R. Beane et al., Nucl. Phys. B768, 38 (2007).

[17] S. R. Beane et al., Phys. Lett. B654, 20 (2007).

[18] S. R. Beane et al., Phys. Rev. Lett. 97, 012001 (2006).

[19] M. Diehl, A. Manashov, and A. Schäfer, Eur. Phys. J. A31, 335 (2007).

[20] M. Diehl, A. Manashov, and A. Schäfer, Eur. Phys. J. A29, 315 (2006).

[21] M. Dorati, T. A. Gail, and T. R. Hemmert, nucl-th/0703073 (2007). 